Gut, 1976, 17, 561-567

\title{
Tissue demonstration of carcinoembryonic antigen (CEA) in ulcerative colitis
}

\author{
P. ISAACSON
}

From the Department of Pathology, Southampton University Hospital, Southampton

SUMMARY An immunoperoxidase technique was used to demonstrate carcinoembryonic antigen (CEA) in sections of proctocolectomy specimens from nine cases of ulcerative colitis in some of which carcinoma and premalignant change had developed. Carcinoma and premalignant change stained positively for CEA in contrast with benign mucosa, whether or not inflammatory dysplasia was present.

Using an immunoperoxidase technique to demonstrate carcinoembryonic antigen (CEA) in formalin fixed, paraffin embedded tissue, Isaacson and Le Vann (1976) have shown that carcinoma can be reliably differentiated from benign mucosa even when the latter shows considerable inflammatory dysplasia. With the use of this technique it was also shown that, in adenomatous polyps, mucosa considered to be premalignant could be discriminated from benign adenomatous glands. In either case, malignant or premalignant mucosa stained strongly for CEA, while benign glands stained either very weakly or not at all. In this study cases of ulcerative colitis were investigated by this technique to determine whether foci of premalignant change could be identified by an increased content of CEA in comparison with surrounding inflamed mucosa.

\section{Methods}

Proctocolectomy specimens from nine cases of ulcerative colitis were examined and their characteristics are summarized in the Table. All the specimens were received fresh from the operating theatre and immediately fixed in $10 \%$ buffered formol saline. An average of 20 sections was examined from each specimen to include, where appropriate, multiple examples of carcinoma, precancerous change, and uncomplicated chronic ulcerative colitis. In deciding whether precancer was present the criteria used were those elaborated by Morson and Pang (1967) and Yardley and Keren (1974). Premalignant change was identified macroscopically as slightly raised plaquelike patches on the mucosa or as sessile villous ade-

Received for publication 12 March 1976
Table Details of cases of ulcerative colitis

\begin{tabular}{|c|c|c|c|c|c|}
\hline $\begin{array}{l}\text { Case } \\
\text { no. }\end{array}$ & $\begin{array}{l}\text { Age } \\
\text { (yr) }\end{array}$ & Sex & $\begin{array}{l}\text { Carci- } \\
\text { noma }\end{array}$ & $\begin{array}{l}\text { Pre- } \\
\text { cancer }\end{array}$ & Other features \\
\hline 1 & 63 & $\mathbf{F}$ & + & + & Multiple carcinomas \\
\hline 2 & 54 & $\mathbf{M}$ & + & + & Multiple carcinomas \\
\hline 3 & 33 & $\mathbf{M}$ & + & + & $\begin{array}{l}\text { Multiple carcinomas } \\
\text { Diffuse premalignant } \\
\text { change }\end{array}$ \\
\hline 4 & 28 & $\mathbf{F}$ & + & - & $\begin{array}{l}\text { Ileorectal } \\
\text { anastamoses }\end{array}$ \\
\hline 5 & 55 & $\mathbf{F}$ & + & - & - \\
\hline 6 & 38 & $\mathbf{M}$ & - & + & - \\
\hline 7 & 34 & $\mathbf{M}$ & - & - & - \\
\hline 8 & 42 & $\mathbf{F}$ & - & - & - \\
\hline 9 & 67 & $\mathbf{F}$ & - & - & - \\
\hline
\end{tabular}

nomatous structures. Microscopically, there was usually a villous pattern (Fig. 2) and epithelial atypia in the form of goblet cell depletion, with hyperchromatic, often stratified, nuclei containing prominent nucleoli. No attempt was made to distinguish between premalignant change and carcinoma-in-situ, although in some instances the cytological atypia was severe enough to suggest malignancy (Fig. 3a). There was usually little accompanying inflammation. The dysplastic changes induced by acute or chronic inflammation (Figs. 5a, 6a) did not give rise to a villous pattern. The glands were more regular but goblet cell depletion was present. The nuclei contained prominent nucleoli but were only moderately hyperchromatic and showed little stratification. There was often a greatly increased number of mitotic figures (Fig. 6a).

Goat antihuman CEA and purified CEA were obtained from the Chester Beatty Research Institute, London, through the generosity of Professor A. Munro Neville. Details of the preparation of the 
CEA and antiserum have been previously described (Isaacson and Le Vann, 1976). Sections were stained for CEA by an indirect immunoperoxidase technique using goat antiCEA followed by peroxidase conjugated horse antigoat immunoglobulin prepared by the method of Nakane and Kawaoi (1974). Serial dilutions of goat antiCEA serum were used to stain a control slide of colonic carcinoma and the dilution one step below that at which the reaction disappeared was used for the study. This dilution varied with each batch of peroxidase conjugated horse antigoat serum but was most often 1 in 200 . Slides were pretreated with undiluted normal horse serum to prevent nonspecific staining and were counterstained with haematoxylin. The details of method used were otherwise as previously described (Isaacson and Le Vann, 1976).

The same case of carcinoma with adjacent normal mucosa was used as a positive control throughout. The characteristic staining of CEA in colonic tissue had previously been shown to be blocked by absorption of the goat antiCEA with purified CEA, and in the study normal goat serum was substituted for goat antiCEA as a negative control.

\section{Results}

Where CEA could be demonstrated in the colonic mucosa it appeared as a thin, slightly irregular line around glandular lumens or over the surface epithelium (Fig. 1). In an occasional cell the cytoplasm showed pale brown staining. Only carcinoma and mucosa showing premalignant change stained positively for CEA. Areas of active or chronic inactive ulcerative colitis were negative even when considerable inflammatory dysplasia was present (Figs. 2, 3, $4,5,6)$.

Where Paneth cell metaplasia occurred these cells stained positively (Fig. 4), as they did in the section of ileum from the ileorectal anastamosis in case 4.

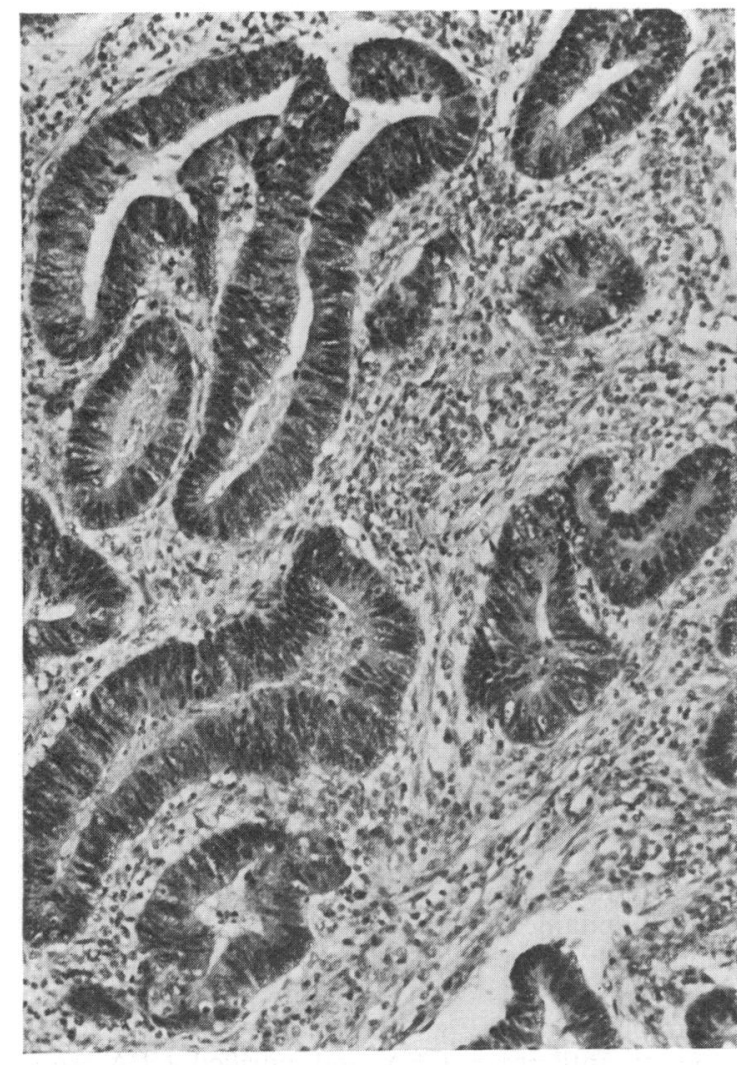

(a)

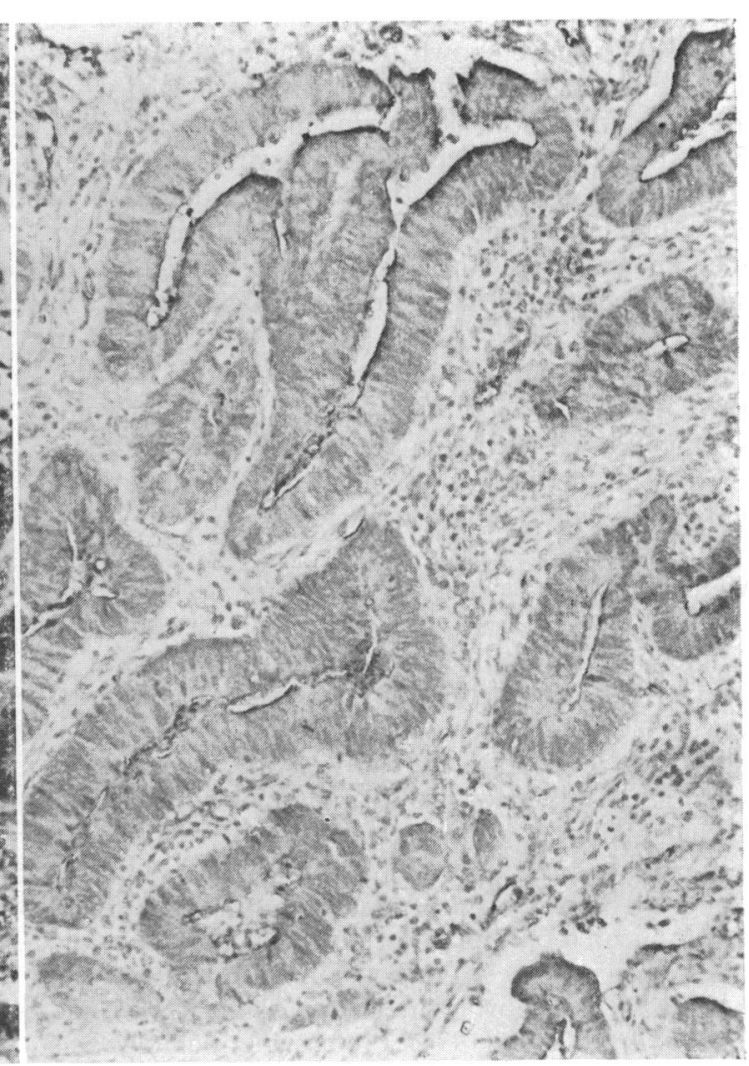

(b)

Fig. 1 Case 4. (a) Rectal carcinoma. $H$ and $E, \times$ 100. (b) Same field stained for CEA which shows up as a slightly irregular dark brown lumenal line. $\times 100$. 


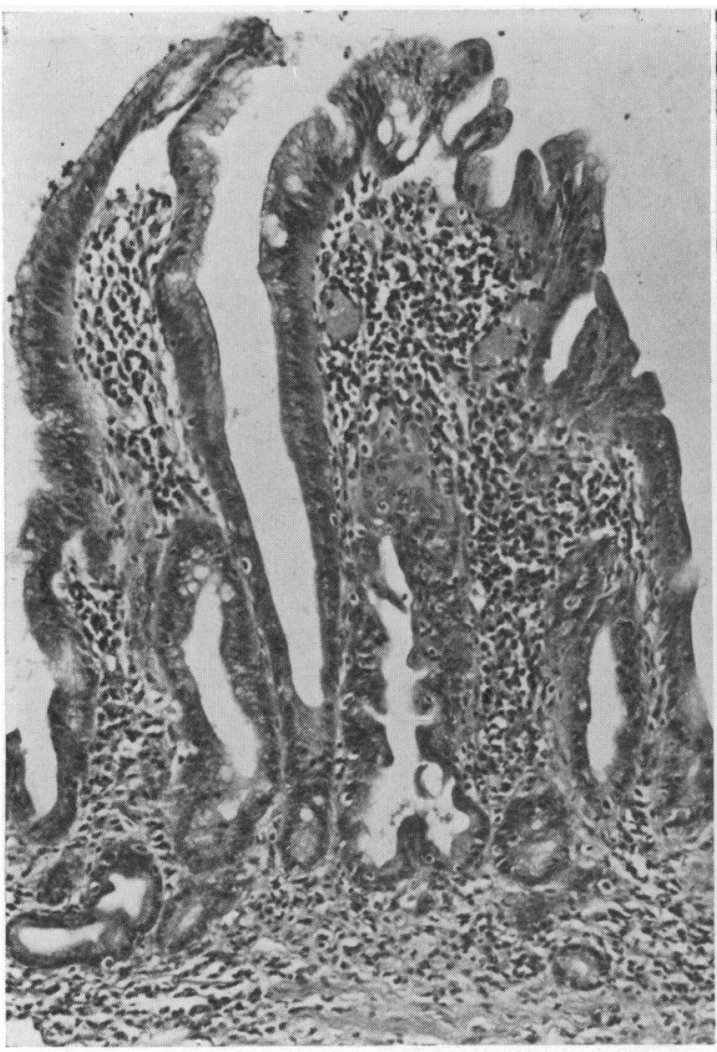

(a)

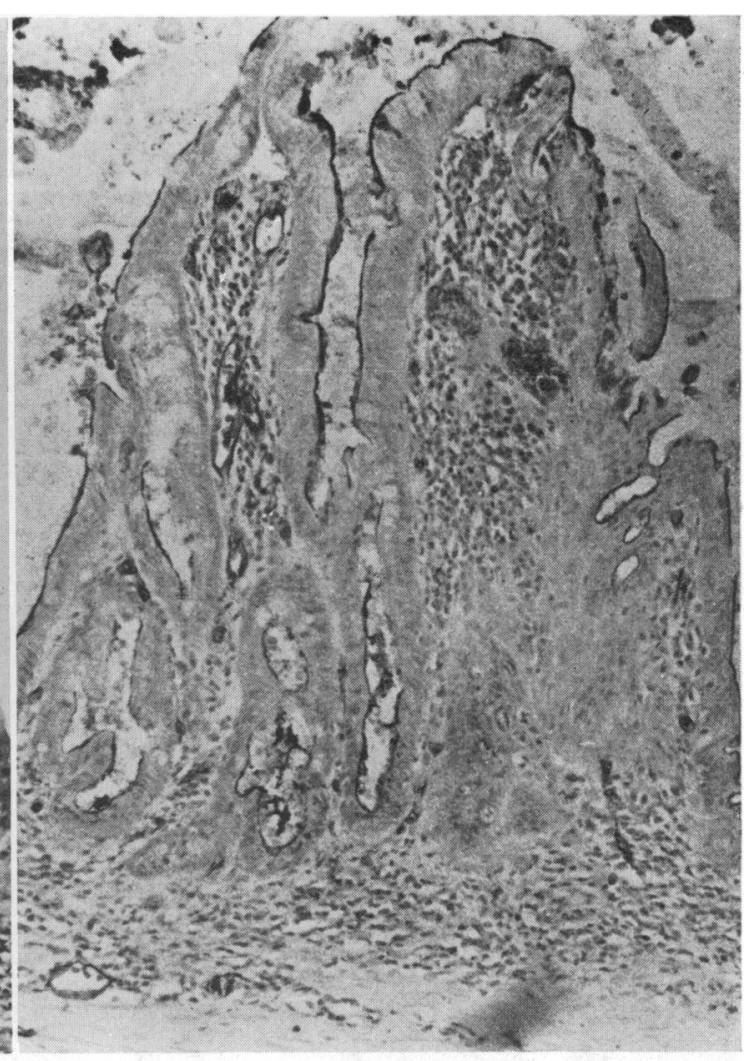

(b)

Fig. 2 Case 3. (a) Mucosa showing premalignant change. Note villous pattern and cytological atypia. $H$ and $E, \times 100$. (b) Same field stained for CEA with positive result. $\times 100$.

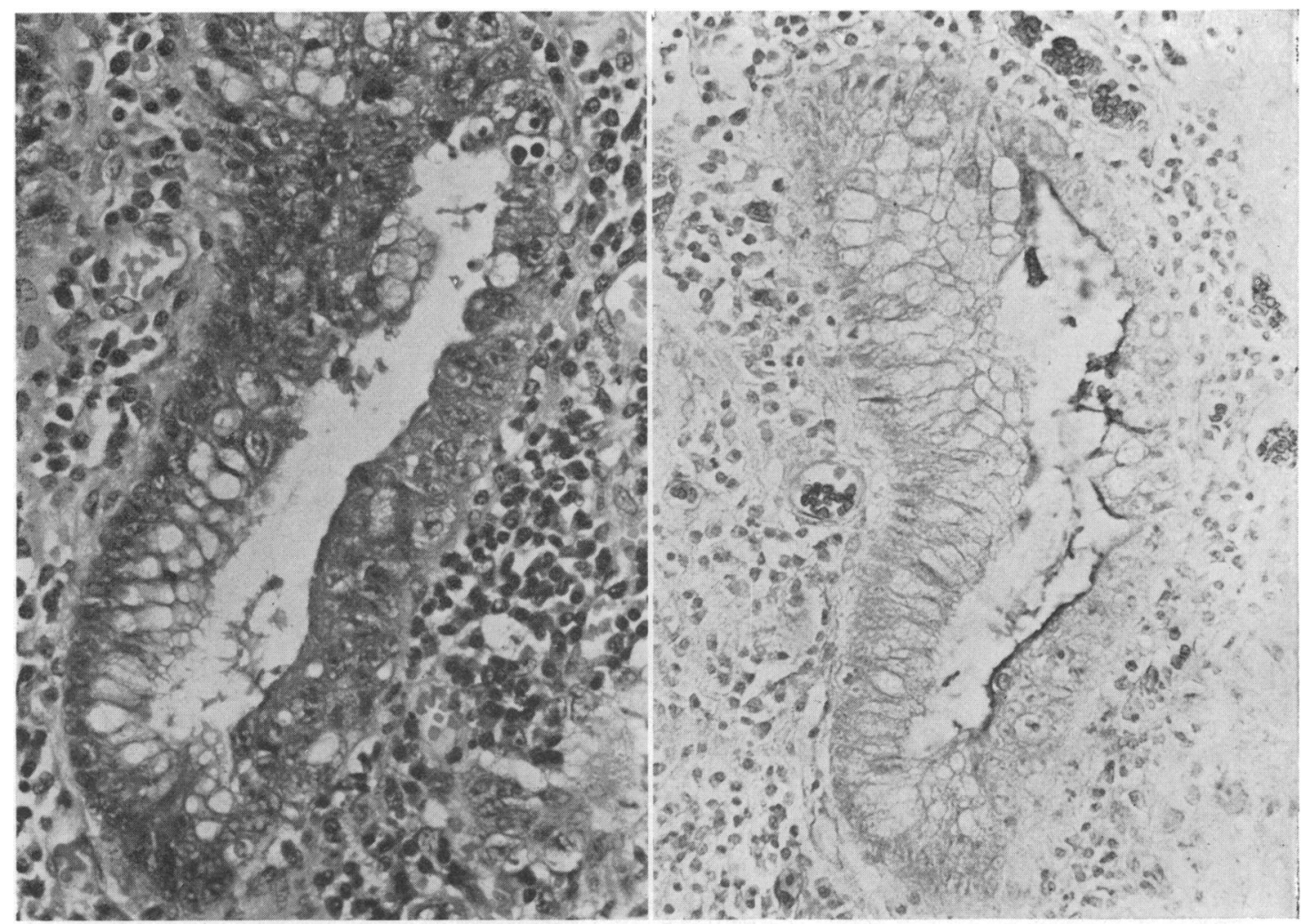

Fig. 3 Case 3. (a) High power view of a gland, part of which shows premalignant change with considerable atypia. $H$ and $E, \times 400$. (b) Same gland stained for CEA which is confined to the portion of the gland showing premalignant change. $\times 400$. 


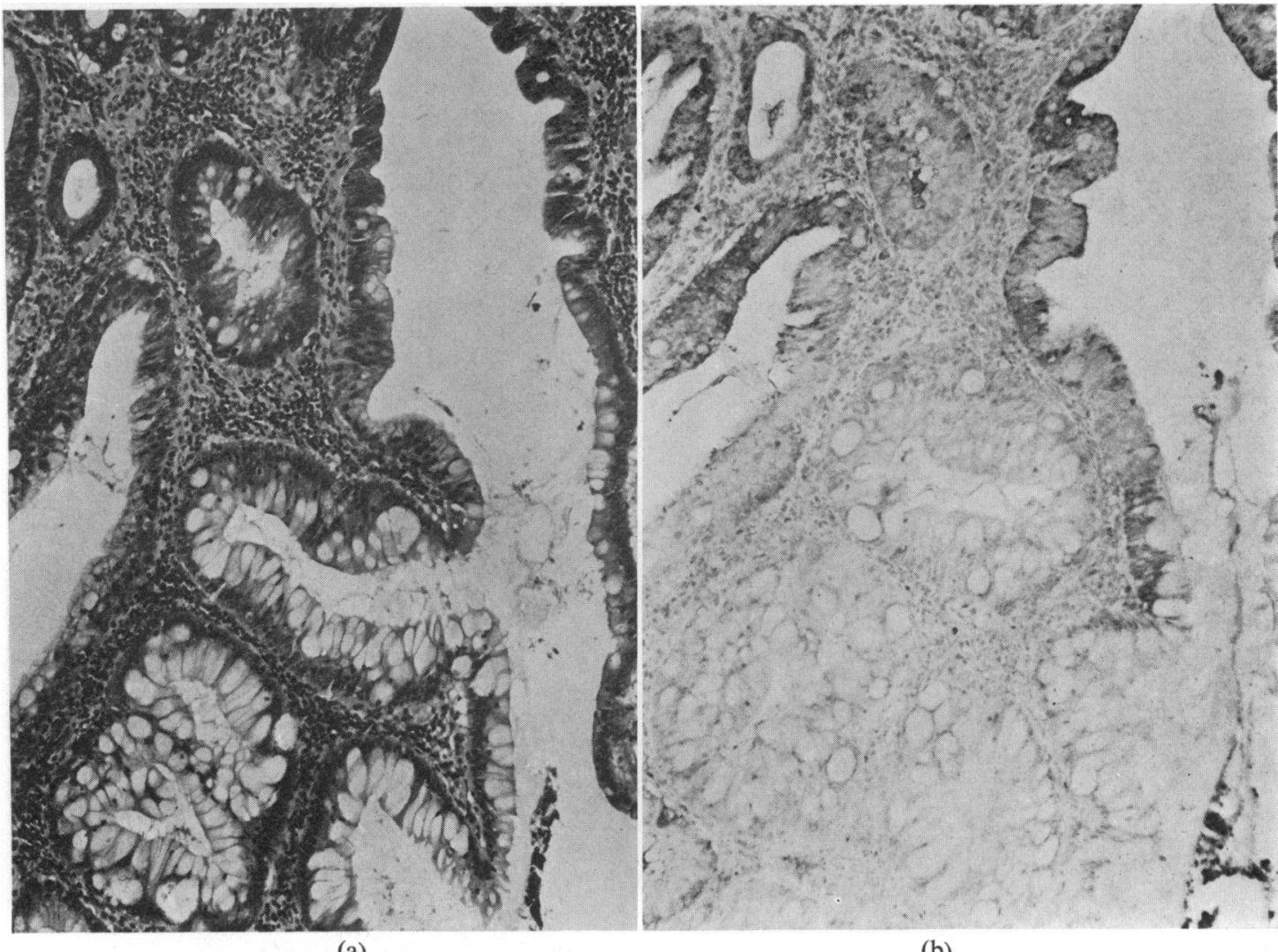

(a)

(b)

Fig. 4 Case 1. (a) Mixture of premalignant and benign glands. Note goblet cells with small basal nuclei in benign glands. $H$ and $E, \times 200$. (b) Staining for $C E A$ shows the antigen related to premalignant areas only. Considerable cytoplasmic staining is caused by many Paneth cells mixed in premalignant epithelium. $\times 200$.

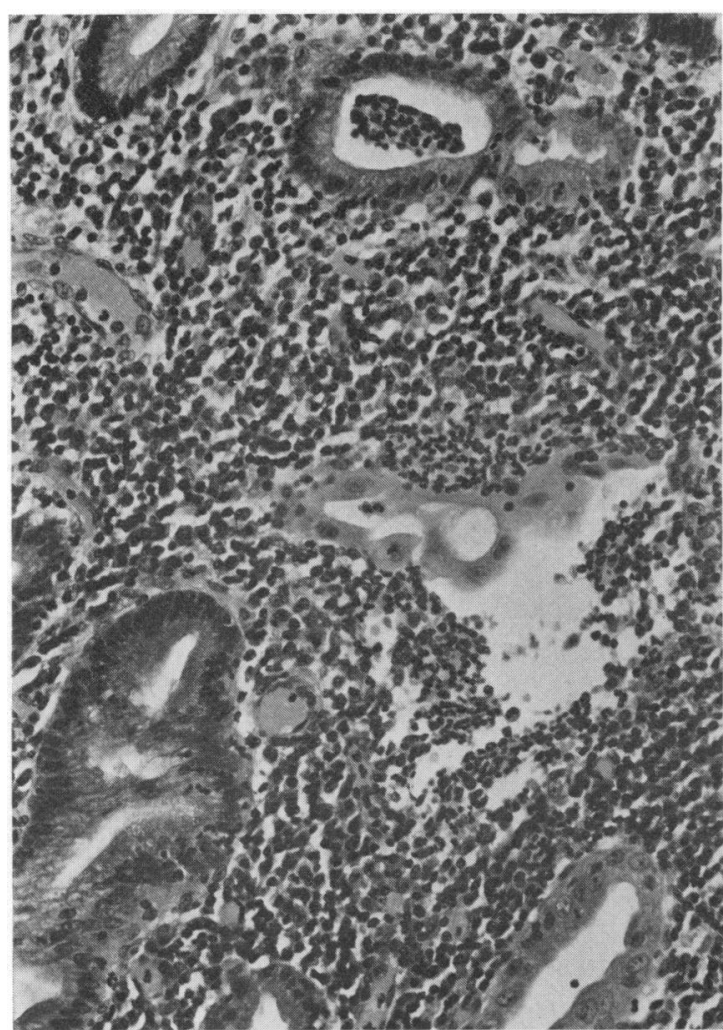

(a)

Fig. 5 Case 4. (a) Inflammatory dysplasia associated with acute inflammation. $H$ and $E, \times 200 .($ b) Negative CEA stain of same field. 


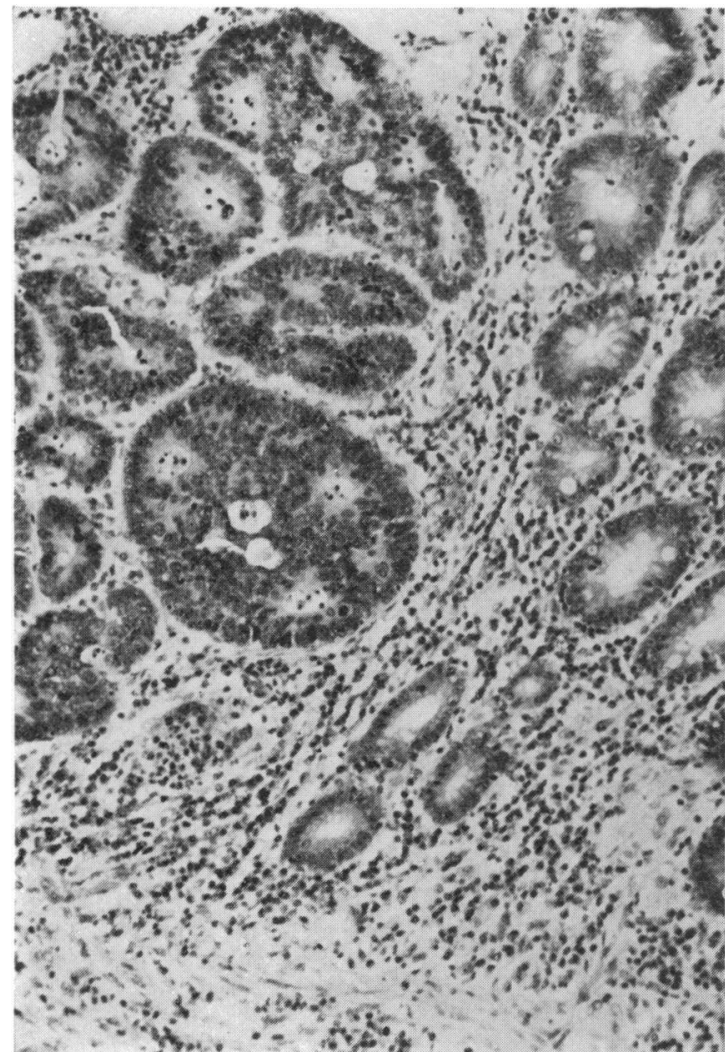

(a)

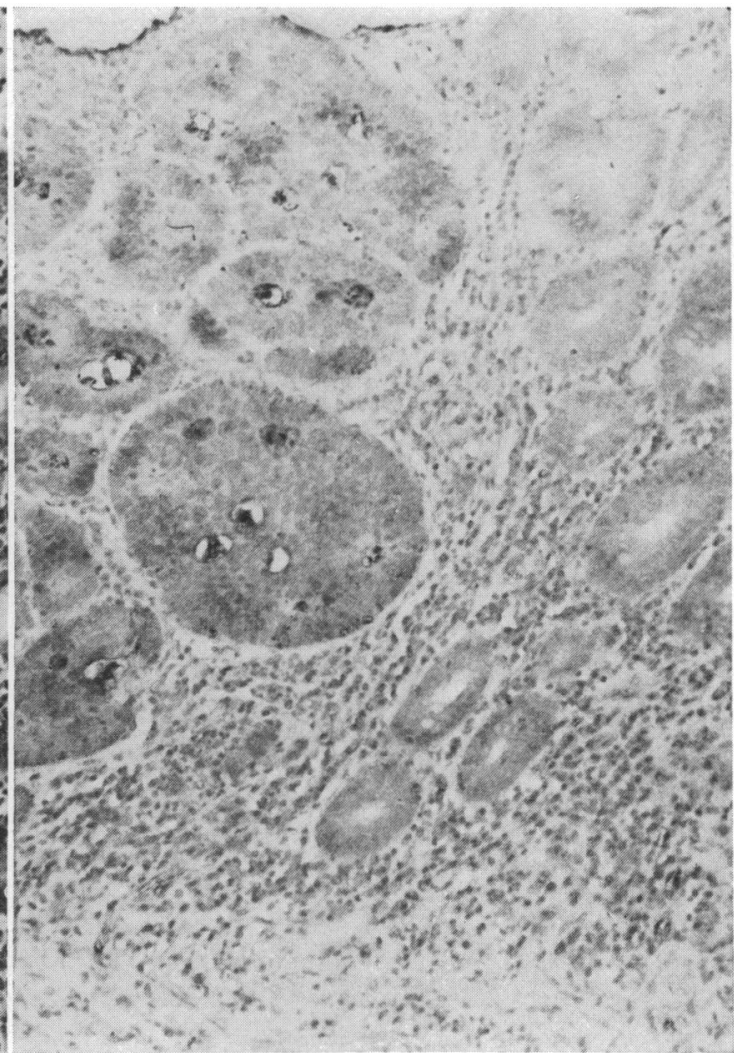

(b)

Fig. 6 Case 5. (a) Mucosa adjacent to carcinoma shows chronic inflammation and dysplasia of glands. Note the numerous mitotic figures. $H$ and $E, \times 100 .(b)$ The carcinoma is positive for CEA while the glands in the inflamed area are negative. $\times 100$.

The ileal mucosa itself was strongly positive. These findings are presently being further investigated and will be the subject of a separate report.

All sections in which normal goat serum was substituted for goat antiCEA were negative.

\section{Discussion}

While the risk of developing carcinoma in a patient with long-standing ulcerative colitis is considerable, the relationship of ulcerative colitis related tumours to the problem of colorectal cancer as a whole is relatively insignificant (Morson, 1974). However, ulcerative colitis provides one of the few instances of well-documented undisputed premalignant change in colonic mucosa and, as such, provides an important model in the search for the early lesion in colorectal cancer.

It is clear that, using this technique, tissue demonstration of CEA in ulcerative colitis is a reliable indicator of premalignant change or carcinoma-in- situ. Previous attempts to show whether the different results in benign and malignant mucosa were due to a qualitative or quantitative difference in CEA production were hampered by nonspecific staining when high concentrations of goat antiCEA (or normal goat serum) were used (Isaacson and Le Vann, 1976). This problem has been overcome by using undiluted normal horse serum as a blocking agent. When high concentrations of goat antiCEA are used CEA can be demonstrated in the normal mucosa of the control slide where it stains weakly and rather patchily (Fig. 7a). This is not surprising, considering the reports of Martin and Martin (1970) and Burtin et al. (1972) who were able to demonstrate CEA in normal mucosa, either by extraction of the antigen or by immunofluorescence. CEA is demonstrated in normal colonic mucosa only when high concentrations of goat antiCEA are used. When the antiserum is diluted staining soon disappears. Malignant mucosa continues to stain positively well beyond the titre at which normal mucosa no longer stains (Fig 


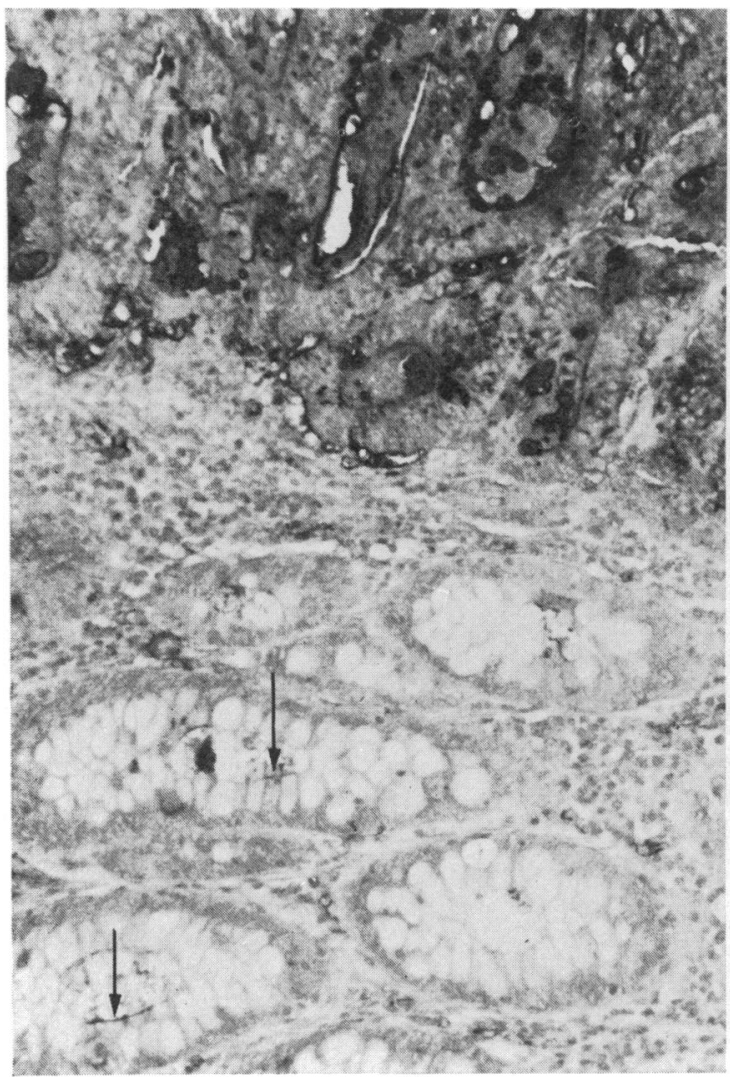

(a)

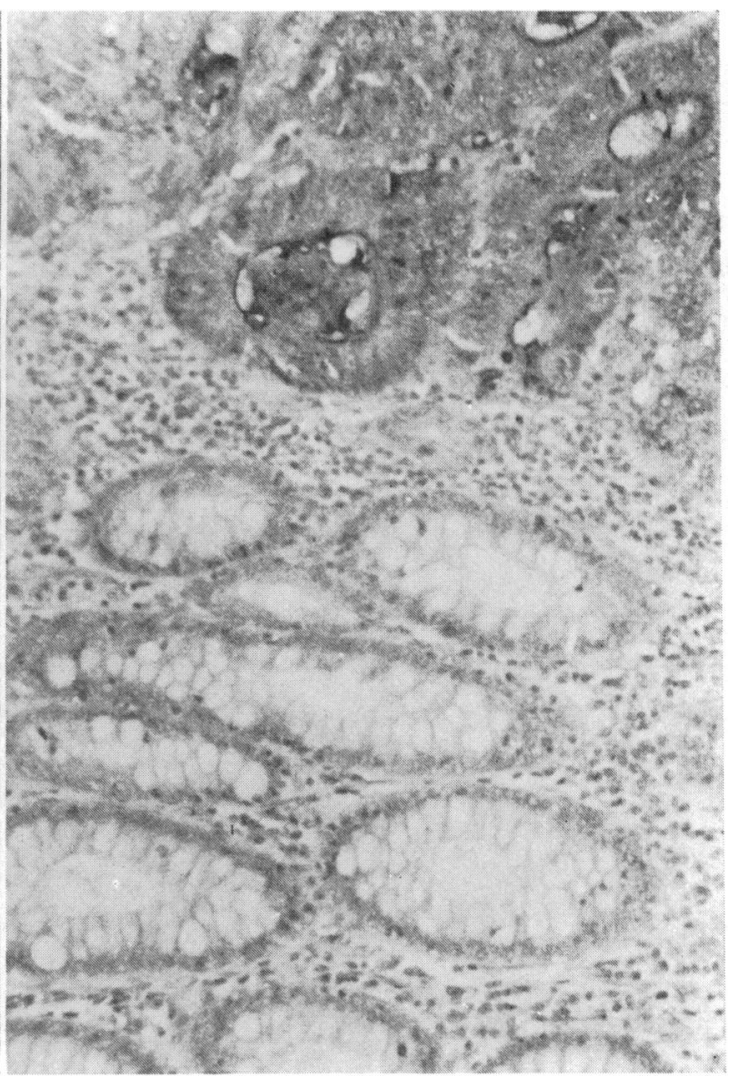

(b)

Fig. 7 (a) Control slide stained with goat antiCEA at a titre of 1 in 10. There is intense staining of the malignant glands and also weak staining of normal mucosa (arrows). $H$ and $E, \times 100$. (b) Same area stained with goat antiCEA 1 in 200. Staining is now confined to the tumour. $\times 100$.

7b). It would appear that, as suggested previously (Isaacson and Le Vann, 1976), it is a quantitative difference in CEA production which allows this method to discriminate between benign and malignant mucosa rather than a specific qualitative difference between the normal cell and the cancer cell. It is possible, however, that in view of the known heterogeneity of CEA some forms of CEA may be more abundant in tumours than others and there may be some cross-reaction between antibodies to various subgroups. Tissue studies with an antiserum to the recently described CEA-S (Edgington et al., 1975), said to be more tumour specific, would be of interest in this respect. Since it appears that a quantitative difference between benign and malignant mucosa is being demonstrated, selection of the correct titre of antiCEA is important if false positive or negative results are to be avoided.

Initial optimism concerning the use of rectal biopsy to diagnose premalignant change in patients with ulcerative colitis (Morson and Pang, 1967) has been tempered somewhat because of interpretive problems caused by inflammatory change (Morson, 1972; Yardley and Keren, 1974). This inflammatory dysplasia may occur in a setting of acute inflammation or severe chronic inflammation (Fig. 5, 6). Staining suspicious biopsies for CEA overcomes this problem and allows a clear distinction between CEA negative inflammatory dysplasia and true precancer which is CEA positive.

There is a difference, of course, between a formal study as described in this paper and use of the method as a routine diagnostic aide. False positives and false negatives may yet occur and histological appearances in themselves should not be ignored. A 'field trial' of the method is in progress and, while preliminary results are encouraging, no doubt instances will arise when morphology and CEA staining do not agree. Careful follow-up will be necessary to solve the dilemma that may be caused by the find- 
ing of morphologically benign, CEA positive areas in a biopsy.

The experience of this laboratory is that almost all examples of premalignant change in ulcerative colitis are obtained from proctocolectomy specimens removed after a diagnosis of invasive carcinoma has been made. The frequency of 'monitoring' rectal biopsies from cases of ulcerative colitis is, however, increasing. Reports that premalignant change in ulcerative colitis is not confined to the rectum (Evans and Pollock, 1972; Teague and Read, 1975) have led to the recommendation that patients with ulcerative colitis be followed with regular colonoscopy and biopsy (Teague and Read, 1975). With the advent of the colonoscope a great increase in the number of small biopsies of suspicious mucosal lesions, from patients with or without ulcerative colitis, is to be expected. Discrimination between benign and malignant mucosa by immunohistochemical demonstration of CEA is potentially a most useful aide for the histopathologist.

I would like to thank Professor D. H. Wright for advice and criticism, Professor A. Munro Neville for the supply of goat antiCEA and purified CEA, and Mrs Mary Judd for expert technical assistance.
References

Burtin, P., Martin, E., Sabine, M. C., and von Kleist, S. (1972). Immunological study of polyps of the colon. Journal of the National Cancer Institute, 48, 25-32.

Edgington, T. S., Astarita, R. W., and Plow, E. F. (1975). Association of an isomeric species of carcinoembryonic antigen with neoplasia of the gastrointestinal tract. New England Journal of Medicine, 293, 103-107.

Evans, D. J., and Pollock, D. J. (1972). In-situ and invasive carcinoma of the colon in patients with ulcerative colitis. Gut, 13, 566-570.

Isaacson, P., and Le Vann, H. P. (1976). The demonstration of carcinoembryonic antigen in colorectal carcinoma and colonic polyps using an immunoperoxidase technique. Cancer. (In press).

Martin, F., and Martin, M. S. (1970). Demonstration of antigens related to colonic cancer in the human digestive system. International Journal of Cancer, 6, 352-360.

Morson, B. C. (1972). Rectal biopsy in inflammatory bowel disease. New England Journal of Medicine, 287, 1337-1339.

Morson, B. C. (1974). Evolution of cancer of the colon and rectum. Cancer, 34, 845-849.

Morson, B. C., and Pang, L. S. C. (1967). Rectal biopsy as an aid to cancer control in ulcerative colitis. Gut, 8, 423-434.

Nakane, P. K., and Kawaoi, A. (1974). Peroxidase-labeled antibody: a new method of conjugation. Journal of Histochemistry and Cytochemistry, 22, 1084-1091.

Teague, R. H., and Read, A. E. (1975). Polyposis in ulcerative colitis. Gut, 16, 792-795.

Yardley, J. H., and Keren, D. F. (1974). 'Precancer' lesions in ulcerative colitis. Cancer, 34, 835-844. 